This item was submitted to Loughborough's Research Repository by the author.

Items in Figshare are protected by copyright, with all rights reserved, unless otherwise indicated.

\title{
Golf coaches' perceptions of key technical swing parameters compared to biomechanical literature
}

PLEASE CITE THE PUBLISHED VERSION

http://dx.doi.org/10.1260/1747-9541.10.4.739

\section{PUBLISHER}

Multi-Science Publishing (now SAGE)

VERSION

AM (Accepted Manuscript)

\section{PUBLISHER STATEMENT}

This work is made available according to the conditions of the Creative Commons Attribution-NonCommercialNoDerivatives 4.0 International (CC BY-NC-ND 4.0) licence. Full details of this licence are available at: https://creativecommons.org/licenses/by-nc-nd/4.0/

\section{LICENCE}

CC BY-NC-ND 4.0

\section{REPOSITORY RECORD}

Smith, Aimee C., Jonathan R. Roberts, Eric S. Wallace, Pui W. Kong, and Stephanie E. Forrester. 2019. "Golf Coaches' Perceptions of Key Technical Swing Parameters Compared to Biomechanical Literature”. figshare. https://hdl.handle.net/2134/21664. 


\title{
Golf Coaches' Perceptions of Key Technical Swing Parameters Compared to Biomechanical Literature
}

\author{
Aimee Smith ${ }^{1,2}$, Jonathan Roberts ${ }^{1}$, Eric Wallace ${ }^{3}$, Pui Wah Kong ${ }^{4}$, Steph Forrester ${ }^{1}$ \\ ${ }^{1}$ Sports Technology Institute, Loughborough University, UK, LE11 3QF. \\ A.C.Smith@lboro.ac.uk \\ ${ }^{2}$ Institute for Sports Research, Nanyang Technological University, Singapore \\ ${ }^{3}$ Ulster University, Ulster, N.Ireland, UK \\ ${ }^{4}$ Physical Education and Sports Science Academic Group, National Institute of Education, Nan- \\ yang Technological University, Singapore.
}

KEYWORDS: golf, qualitative analysis, coaching, biomechanics, perception.

\begin{abstract}
Assessing a coach's technical knowledge of a sporting technique can reveal measureable biomechanical parameters associated with a successful performance. Therefore, the purpose of this study was to identify the key technical parameters that golf coaches associate with a successful golf swing. Sixteen high-level golf coaches were observed coaching a highly skilled golfer after which they participated in a semi-structured interview regarding their technical analysis of the golf swing. The data were inductively analysed and five intrinsically linked key technical parameters were identified: 'Posture', Body Rotation', 'Arm and Wrist Action', 'Sequential Movement and Body Segments' and 'Club Motion'. The parameters Posture and Body Rotation were further sub-categorised and compared to the existing biomechanical literature whereby gaps in knowledge were identified. The results of this study can be used to guide future golf biomechanics research and coaching technologies..
\end{abstract}

\section{INTRODUCTION}

An important aspect of coaching involves the analysis of sporting movements in order to effect a change in performance [1]. Coaches are required to make accurate and reliable observations of a performer's movement patterns and subsequently guide their performance towards a more optimal or ideal technique through appropriate coaching sessions. To do this, it is assumed that coaches have a well-developed internal model of a technically correct performance against which they compare the performers technique [1]. The formation of such a model is proposed to be influenced by several aspects including a coach's technical knowledge, which includes their biomechanical understanding of the technique [2]. The extent of a coach's technical knowledge is an area often not investigated in research studies and this has led to the development of the coaching-biomechanics interface [2].

The coaching-biomechanics is a term that conceptualises how coaching can be informed from a biomechanical perspective and inevitably aims to bridge the gap between underlying biomechanical parameters and coaching in- formation (Kerwin \& Irwin, 2007). The coachingbiomechanics interface begins by examining the content of a coach's technical knowledge regarding a performer's technique. The information gleaned from such insights, through interviews or observations, is then converted into measureable biomechanical parameters that may be directly related to a successful performance. This information can provide new insights into the technique, reinforce previously accepted coaching ideas, enhance a coach's technical understanding and assist in optimising performance [2]. Assessing an expert coach's technical knowledge and the sources of such knowledge has been conducted for sports such as gymnastics and sprinting and has provided information to guide future biomechanical studies, which were largely uninvestigated in the literature [3-5].

Sherman et al. [1] reported that regardless of golf coaching ability, golf coaches seemingly individualised their perception of key technical swing parameters based on the golfer's expertise and physique. The key technical parameters being analysed, however, were pre-defined by 
the authors rather than based on the content of the coaches' current technical knowledge. Other studies have attempted to understand how expert golf coaches learn and the sources of this information [6]; however, this has not subsequently been related to the content of their technical knowledge of the golf swing. Indeed, despite numerous golf instructional books, there have been few, if any, scientific studies, which have investigated the content of a golf coach's technical knowledge. Adlington [7] provided a review of ideal swing technique and biomechanics aimed at reducing the risk of injury. Similarly, Hume et al. [8] reported key technical parameters based on a review of the current golf biomechanical literature. Neither review, however, investigated the golf coaches' perceptions of the key technical parameters based on the concept of the coaching-biomechanics interface.

In studies investigating coaches' perceptions, no attempt is made to assess the validity of the coaches' views and it is acknowledged that their views may be based on personal experience rather than underpinned by scientific evidence. Nevertheless, high-level golf coaches have a vast amount of practical experience with regards to developing a golfer's swing and therefore can offer a valuable perspective. Studying the methods, beliefs and technical knowledge of high-level coaches with a view to provide new insights and hypotheses that can be explored scientifically is a recognised approach [9]. Thus, using the high-level golf coaches' technical knowledge to help inform future biomechanical studies appears a sensible route to explore.

The purpose of this study was to use the principles of the coaching-biomechanics interface to identify the key technical parameters that high-level golf coaches associate with a successful golf swing. These parameters would then be used to identify similarities or differences to current golf biomechanical literature to guide future biomechanical research. The term successful was used to define a golf swing that resulted in the intended shot direction and displacement, as was defined by the golf coaches during the qualitative study. The purpose of this study would be achieved by addressing two objectives. The first objective was to qualitatively analyse the coaches' responses regarding the key technical parameters of a successful golf swing. The second objective was to compare, and place, the key technical parameters within the current golf biomechanical literature. The results will allow technical parameters to be investigated in future biomechanical analysis, which may help to reinforce existing coaching knowledge, provide new insights to assist future technique development, enhance understanding of the golf swing and direct new technologies to aid golf coaching.

\section{METHODS}

\section{PARTICIPANT SELECTION}

Sixteen high-level golf coaches participated in the study. A minimum sample size of fifteen golf coaches was initially deemed appropriate given the time and resources available. After interviewing the fifteen coaches it became apparent that a 'saturation' stage had been reached and therefore another coach was recruited to confirm saturation and the study was subsequently terminated. The participants were aged $24-51$ years $($ mean $=39.0$ years; $s=7.6$ years) and had an average of 18 years of golf coaching expertise ( $s=8.2$ years). Several criteria were specified to identify golf coaches with an appropriate level of technical knowledge. Firstly, the coach had gained at least a Level 3 Professional Golf Association (PGA) qualification, with Level 4 being the pinnacle of current golf coach education in the UK. Secondly, the coach had five or more years of coaching experience and was currently still coaching. Finally, the coach needed experience of coaching an elite golfer, for example a tour level golfer or international golfer. Of the coaches who participated fifteen had coached a golfer that had played on a either an amateur or professional tour and one had coached an international level golfer. In addition, all coaches that participated were scratch golfers and several had played golf to a high level before pursuing a career in golf coaching. Previous research studies investigating a coach's technical knowledge have used similar criteria when defining coaching expertise [3,10,11]. All golf coaches and golfers observed during the technical sessions gave their informed consent and ethical clearance was obtained from Loughborough University Ethical Advisory Committee.

\section{DATA COLLECTION}

A combination of observations followed by interviews were used to determine the golf coaches' perceptions of the key technical parameters of an elite golf swing. Conducting interviews after the participant has been observed can allow more in depth exploration of the key themes identified during the observation [12] and help to inform the focus of proceeding interviews [13].

\section{OBSERVATION}

An overt observational style was adopted in a field setting where a typical technical coaching session, led by the golf coach, would take place. A technical coaching session was defined as a session where the golfer would use a driver or long iron and the focus was on the full golf swing. The golfer being coached was requested to be of the highest standard accessible to the coach at the time of the observation, for example an elite golfer. The coaching sessions lasted between 45 and 120 minutes. A standard video camera (Panasonic, Japan) was used to obtain a record of the coaching session. The video camera was positioned at an appropriate distance from the coach and golfer so that the session could be visually and audibly recorded whilst not interfering with the coaching session. In addition, an observer stood near the technical coaching area to record comprehensive field notes of the coaching session. The terms and phrases used by the coach were noted and used during proceeding interviews. An observation guide was used to organise the field notes into four sections detailing the structure of the session, coach behaviour, technology used and technical analysis of the golf swing. 


\section{INTERVIEWS}

Following the observation, a semi-structured interview was conducted with the coach. This approach allows interviews to be partially guided by observational findings whilst still remaining systematic across coaches by using guided unambiguous questions. The interviews were conducted at the same location as the coaching session; therefore increasing the level of comfort for the coach and the probability of attaining high-quality information [14].

An interview guide, divided into two sections, was designed and implemented to provide a basic structure to the interview enabling continuity and comparability between interviews [15]. The coach was given a brief introduction to the interview purpose and was instructed to answer all questions in relation to an elite golfer's swing. The first section focussed on the structure of the technical coaching session with information gleaned about their coaching behaviour, for example the position from which they observed the golfer and their use of technology. The second section focussed on their perception of the technical aspects of an elite golf swing. Each section began with an initial open-ended question, followed by further questioning to explore the coaches response in more detail as to their precise meaning. The questions asked were unambiguous and did not force the responses from the coaches, which was confirmed by the feedback given by coaches following the interview process [15]. Any information gleaned from the observations that were not commented on by coaches during initial questioning were also introduced and probed with further questioning. The interviews lasted between 30 - 45 minutes and were recorded using a Dictaphone (Zoom, Japan) from which typed transcripts were produced for data analysis.

\section{DATA ANALYSIS}

Based on grounded theory, an inductive approach to qualitative data analysis was used to identify the golf coaches' perceptions of key technical parameters [12]. This approach allowed important technical parameters to emerge from the data and has been successful in studies of similar purpose, for example, when exploring elite sprint coaches' knowledge of sprinting [3,11]. The QSRNVivo (QSR International, Australia) qualitative analysis software was used as it allowed all sources of data, for example video and audio, to be collated within a single project.

Following transcription of each interview, it was important to become grounded in the data in order to begin organising the data into meaning units based on the content, in a process known as coding [16] (Figure 1). Transcripts were coded line-by-line by the principal researcher (AS), which involved highlighting quotes into meaningful units of data, which represented an event, object or action/interaction [17]. Excerpts of video, captured during the observation, were also coded in this way.

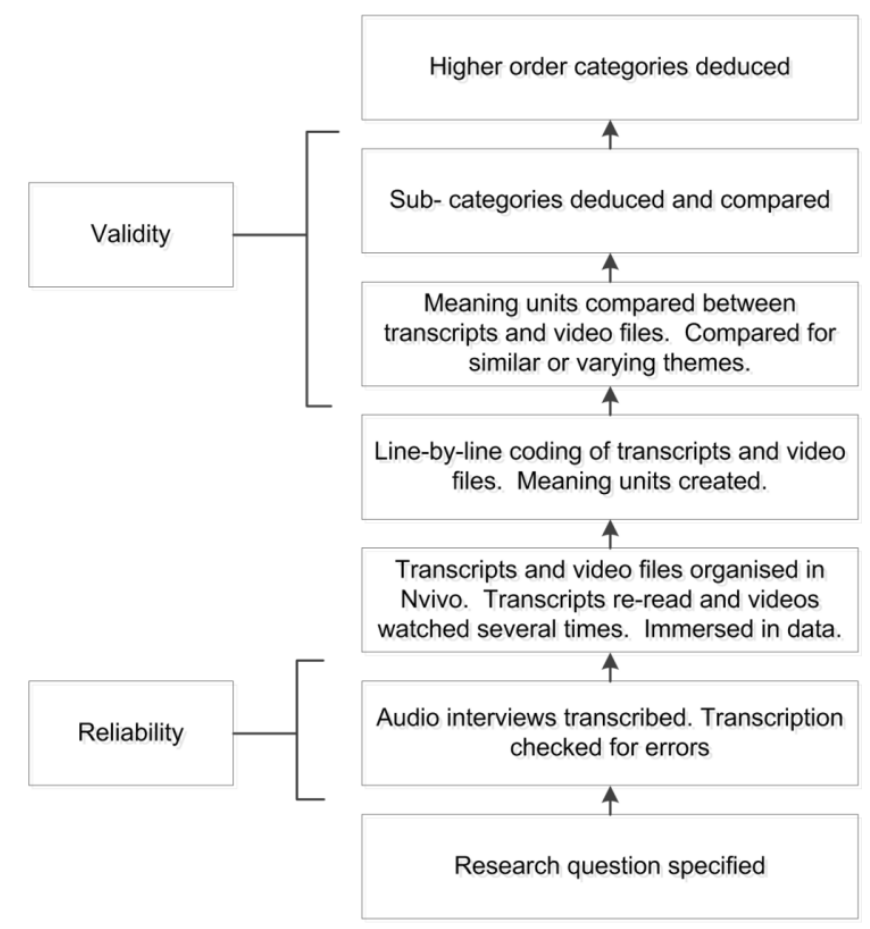

Figure 1. Inductive qualitative data analysis approach used in this study based on grounded theory [12]

The meaning units were compared for similarities and differences in themes. Those found to have similar themes were grouped together at a higher level into subcategories. If sub-categories also shared a common theme these were grouped together and became branches to an overall higher order category. This process was continued until there were as few unassignable themes as possible. Themes that were unassignable were either disregarded or kept if regarded important. To refine the coding hierarchy, the terms used for meaning units were re-examined and those terms deemed to serve a similar analytical purpose were given a single meaning unit title. The constant comparison of units ensured a close connection between codes and the data and provided a check for coding consistency [17]. Furthermore, the researcher revisited a single transcript and made notes on the themes and then compared this to the original coding to ensure coding was consistent and accurate. A second researcher was also given several excerpts of a coach's transcript from the interview and was instructed to carry out lineby-line coding to identify their own meaning units. The meaning units identified by the second researcher were then compared to the original meaning units to ensure that the most appropriate interpretation of the data had been achieved.

The outcome of this analysis resulted in several higher order categories, sub-categories and associated meaning units, which represented the golf coaches' perceptions of the key technical parameters that were associated with an elite golf swing. 


\section{RESULTS AND DISCUSSION \\ OVERVIEW}

Five higher order categories emerged from the inductive analysis as to the key technical parameters of the golf swing which were 'Club Motion', 'Posture', 'Body Rotation', 'Sequential Movement of Body Segments' and 'Arm and Wrist Action'. The higher categories, sub-categories and meaning units for the five key technical parameters can be found in Figure 2. For the purpose of this paper, only the golf coaches' perceptions of Posture and Body rotation are presented in detail and compared to the current golf biomechanical literature. Posture and Body rotation were the most common themes discussed by golf coaches and were therefore chosen to be reported in this paper.

The results and discussion are divided into three sections, firstly, the definition of the stages of the swing identified by golf coaches will be discussed. The second section discussed the key technical parameters within the golf coach's context, including example quotes, in order to develop an understanding of the coaches' perceptions of the key technical parameters. Finally, the current golf biomechanical knowledge related to stages of the swing, posture and body rotation are discussed and finally compared to the coaches' perceptions. Future research directions for biomechanical analysis are then suggested.

\section{STAGES OF THE SWING}

Many golf coaches referred to the key technical parameters of the golf swing at specific stages throughout the swing:

First, I would look at address position...then into the backswing to the top, then the start down, moving into impact and from impact to arms straight is follow through, then follow through to finish. So I'd analyse each bit. [Q1 (Quote 1)]

'Address' and 'Impact' were viewed as the most critical stages of the golf swing. The address or set-up included the ball position, the golfer's alignment to the chosen target and their body position before the golf club was swung. From the observations, all golf coaches would define a target to which the golfer would aim their golf shots during their coaching session. In addition, some coaches paid particular attention to where the golf ball was positioned relative to the golfer. An incorrect ball position, relative to the golfer's stance at set-up was linked to changes in a golfer's body movements.

If we're dealing with an elite golfer [a centred strike] should be very easy to attain. Usually something is misaligned in the set up or ball position...[Q2]

Ensuring the key technical parameters were correct and repeatable at set-up was important for coaches and a parameter most often referred to at this stage was 'Posture'.

Following Address, some coaches referred to the 'Backswing' phase, which culminated when the golfer reached the 'Top of the Backswing'. The Top of the Backswing was defined in two ways by the coaches; the first definition was when the golfer felt they could not rotate their 'shoulders' any further and the second definition was when the club had stopped and then begun moving again.

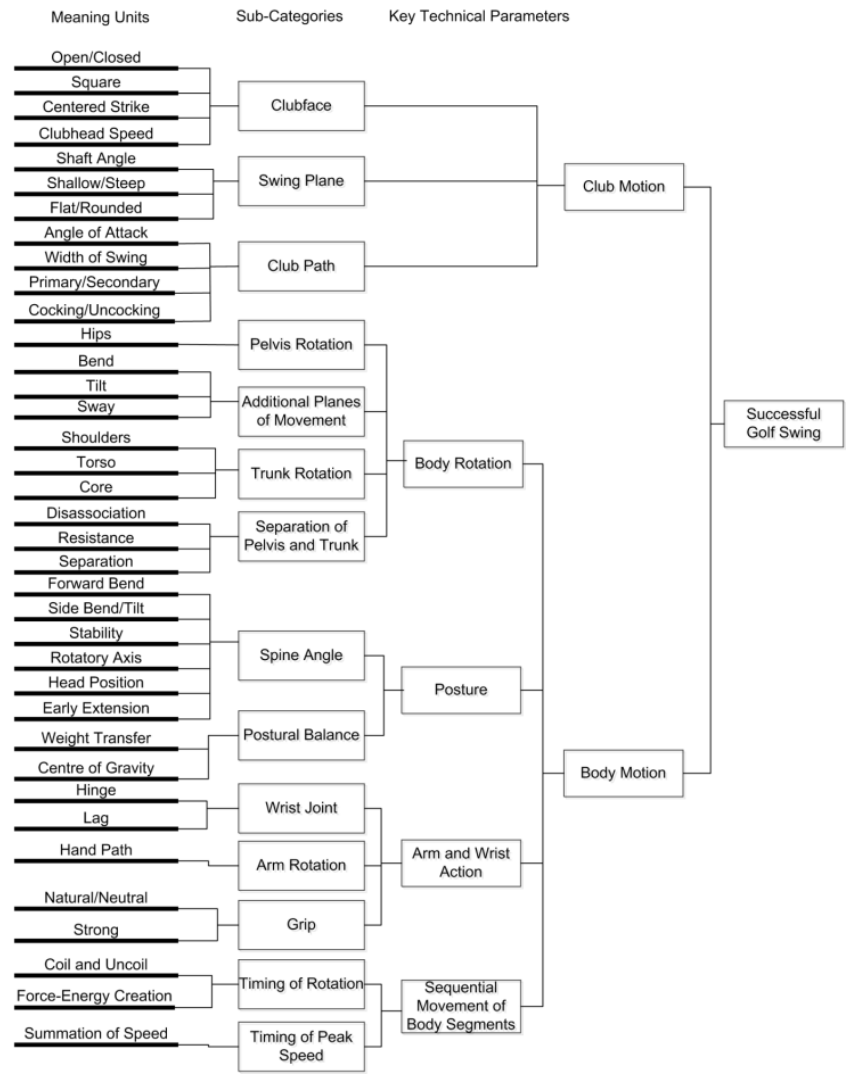

Figure 2. (a) Diagram showing the key technical parameters including sub-categories and meaning units.

Body rotation and Posture were often referred to in conjunction with the backswing and for one coach, creating a Top of the Backswing position through these parameters enabled the rest of the swing to work efficiently:

If we can get [the golfer] in a correct position at top of the backswing, everything reacts off the back of that...it's efficient. [Q $\left.Q_{3}\right]$

Proceeding Top of the Backswing, the coaches spoke about the 'Downswing' phase, which was initiated by an 'Initial Downswing' or transition phase when the club began moving and ended at impact. For one coach, the Initial Downswing movement was considered as the most critical point of the downswing and ended at Impact:

I want the initial movement [in the downswing] to be good and once we're on plane there, it is very difficult to get off that plane. [Q4]

Impact was regarded as a crucial stage during the golf swing. For one golf coach Impact was:

The transfer of energy... between club and ball...that is what creates ball flight... where the clubhead is at impact and how your club moves through [impact]. [Q5]

The coaches discussed all of the key technical parameters in relation to this stage. Some coaches also believed that the 
Impact position of the club would inevitably be the same across golfers as each golfer would be striving for the same clubhead parameters (e.g. centred strike).

Finally, the coaches spoke about the 'Follow Through' and 'Finish Position' as the point where the arms were straight and when the club finally stopped respectively. The Follow Through and Finish Positions were not widely discussed in relation to the key technical parameters, perhaps due to many coaches only interested in the swing through to Impact.

Whilst coaches acknowledged the need to break the swing into stages in order to technically analyse key technical parameters, some emphasised the need to examine the 'Whole Swing'.

I think there are crucial elements, like set-up, impact...so I do break down elements of it but I try and [have] drills...that help promote motion, movement, rhythm and tempo...I don't like to see players who are transfixed about getting clubs in position, it's a movement. [Q6]

The coaches believed that tracing the golfer's movements throughout the swing was equally, if not more important, than solely focusing on specific stages of the golf swing. In addition, one coach highlighted a potential downfall with current biomechanical analysis.

The problem with a lot of the [biomechanical analysis] systems [are] they generally track what it is like at the start or the end of the movement...but how has that happened...is more important. [Q7]

In biomechanical literature, the most notable stages of the swing used in analysis are top of the backswing (TB) and impact (IMP). Other swing events used for analysis include; takeaway (TA), mid-backswing (MidBS), late-backswing (LateBS), acceleration (Acc), mid-downswing (MidDS), 40 ms to impact (40 ms), impact (IMP), mid follow through (MidFT) and end of follow through (FT). However, there are discrepancies between studies when defining some stages of the swing. For example, TB has been defined in several ways: club reaching maximum rotation [18]; club reaches most lateral point before changing direction [19,20]; maximum pelvis rotation [21] and maximum upper torso/shoulder rotation [22]. The discrepancies in defining the swing events can affect interpretation of some results, such as swing time.

Recent studies recognised the limitation with data analysis at key events for biomechanical analysis as a large majority of the continuous data signal is unaccounted for during analysis [23]. Hence, functional data analysis techniques have been employed to detect patterns within a continuous data signal $[24,25]$.

In summary, the discrepancy in defining some stages of the swing (e.g. TB) was evident in both the coaches' perceptions of stages of the swing and the literature. The coaches' perceptions of the most important swing events [Q1] were also not fully supported by the literature as some studies did not consider TA and the backswing to be important points when analysing the golf swing. Furthermore, the need for more advanced biomechanical analysis methods, which can account for the whole swing was noted by some coaches [Q7] and is currently lacking in the literature.

\section{POSTURE}

Fourteen coaches identified 'Posture' as a key technical parameter of the golf swing. Posture was typically discussed in terms of a golfer's body position at set-up and their ability to control or maintain their posture throughout the swing.

On further clarification, two sub-categories of posture were identified: 'Spine angle' and 'Postural Balance'. Spine angle often referred to the degree of 'Forward Bend/Flexion' of the trunk or spine to the pelvis and was viewed as a 'Rotatory Axis'. For one coach Posture was defined as:

...having the correct amount of forward bend to the pelvis and torso, keeping the lumbar and thoracic as neutral as possible so bending forward from the hips, not so much from the knees, or rounded back. [Q8]

Typically, coaches observed a golfer's Spine angle from a side on position whilst some also made reference to the degree of tilt in the upper torso, which was viewed from a front on position.

\section{...it's important to have good basic foundations from \\ a set up point of view...good forward bends and good tilts...when we're talking about tilt we'll look at side bend, so looking at the golfer face [Q9]}

It was considered important that golfers established and maintained their Spine angle from set-up throughout the swing to act as an axis, which the golfer could rotate around. Maintaining a stable Rotatory Axis was essential to creating powerful, efficient and consistent club motion and was sometimes valued above other key technical parameters such as the degree of body rotation. As one coach stated:

[The golfers] that are more efficient [and]...powerful are the guys that maintain a good centre and rotate around it...not necessarily making massive rotations...it's about maintaining those postures...to reduce injury and to allow the club to get back to the golf ball more consistently. [Q10]

Often a loss in Spine angle towards impact was described as 'Early Extension' or 'Hip Thrust', which was linked to golfers with limited rotation through impact and was viewed as a detrimental movement in the swing.

Early extension has to do with rotation...you have a lot of people who come into impact and stop rotating so they're not carrying the ball...they stop and stand up.[Q11]

The coaches also defined a golfer's Posture as creating a balanced body position, which was categorised as 'Postural Balance'.

[Posture is when the] body is in a balanced position that enables the club to get moving efficiently and effectively around the body...if somebody's weight gets too much on the heels it's...difficult to...get the correct pitch of the shoulders in the backswing. [Q12]

One coach defined Postural Balance statically at set-up as positioning the 'Centre of Gravity' correctly or creating balance points in a repeatable manner.

The reason for posture...is to develop the two key balance points...the sternum and belt buckle...getting the centre of gravity right and getting the 
balance points right...the key then is body motion...are you able to rotate. [Q13]

Some coaches referred to Postural Balance as tracing the golfer's 'Weight Transfer' from set-up through the golf swing and having good interaction with the ground. A balanced set-up position allowed the golfer to be able to rotate more effectively with improved dynamic Postural Balance.

Balance...would be your most natural position at address where you...interact with the ground best and shouldn't have to...make a compensation to create an athletic movement. [Q14]

If my hips, my chest, my arms, my hands move in the wrong order or out of posture, then I'll be in poor dynamic balance and not really able to bring the power down or centre the strike. [Q15]

In contrast to the importance placed on posture by golf coaches it has received relatively little attention in the golf biomechanical literature. In the biomechanical literature, posture has been described in terms of the position of the body relative to the vertical, which shall be referred to as postural kinematics and includes variables such as trunk flexion and lateral bend [26]. Hume et al.'s [8] review of key biomechanical parameters of the golf swing, included establishing and maintain posture from set-up and throughout the swing. An optimal posture at set-up was defined as trunk flexed approximately $45^{\circ}$ and tilted approximately $16^{\circ}$ from the vertical and was deemed ideal for generating power and maintaining control during the golf swing [8]. Chu et al. [27] reported minimal change in the golfer's trunk flexion from TB to IMP $\left(\sim 2-3^{\circ}\right)$ and suggested that this angle should remain constant throughout the swing to allow the trunk rotation to be maintained on a plane. In contrast, McTeigue et al. [28] claimed that trying to maintain constant trunk flexion could cause excessive lateral bending and backward bending at TB, which may cause injury.

An increase in trunk right side lateral bend (mean $8.6 \pm$ $6.0^{\circ}$ to $14.4 \pm 6.5^{\circ}$ ) from Acc to IMP has been postulated to create an upward angle of the club path towards impact [27]. An increased angle of the club path (i.e. attack angle) at IMP has been reported to positively influence driving distance [29]. Chu et al. [27] also suggested that trunk lateral bend should occur in a short period of time prior to IMP as early lateral bending could restrict trunk rotation. McTeigue et al. [28] also commented on the greater increase in trunk right lateral bend angle towards IMP of tour players compared to amateur golfers. However, there were large differences (of approximately $16^{\circ}$ ) in the magnitude of trunk lateral bend angle at IMP to the values of Chu et al. [27].

Postural kinematics have often been reported as $2 \mathrm{D}$ trunk angles obtained from motion analysis systems (e.g. angle between the vertical global axis and vector created between shoulder and pelvis markers) [27,30] and electromagnetic systems based on a single rigid trunk segment [28,31]. Differences in the methodologies including the trunk definitions used could explain differences in the magnitudes of postural kinematics reported in the literature.

Posture is also regarded as a dynamic variable of balance to prevent falling. Two independent measures are whole body centre of gravity (COG) (i.e. weighted average of the COG of each body segment in $3 \mathrm{D}$ space) and centre of pressure $(\mathrm{COP})$ (i.e. the $2 \mathrm{D}$ point location in the horizontal plane where the resultant of all ground reaction forces act) [26]. The relationship between COG and COP measures have been used to investigate static and dynamic balance control [32]. In golf, Ball and Best [33] presented two distinct COP styles, 'front foot' and 'reverse foot', defined using the percentage of COP $(\% \mathrm{COP})$ between the front (i.e. foot closest to target = o\%) and back foot (i.e. foot furthest away from target = $100 \%)$. Front foot style was defined as a balanced position at TA (\%COP 57\%), moving to the back foot in the backswing (\%COP 21\%) then onto the front foot at IMP (\%COP 91\%) [33]. The reverse foot style was characterised by players positioning COP towards the front foot in the early downswing (\%COP 61\%) but then re-positioning COP towards midstance at IMP (\%COP 53\%) and towards the back foot in the FT (\%COP 40\%). No statistical differences were reported in clubhead velocity between front and reverse foot style golfers [33], but more recently differences in clubhead velocity within styles have been observed [34]. Front foot style golfers with a greater range of COP movement and increased rate of COP movement to the front foot in the downswing were associated with higher clubhead velocity. Reverse foot golfers with higher clubhead velocity had COP measures near mid-stance and greater rate of COP towards the back foot at IMP.

The studies of Ball and Best [33-35] do not account for COP between the toes and heel (i.e. anterior-posterior direction) or linked it to COG position, which could identify further balance control strategies. Burden et al. [36] is the only study to report the COG path throughout the golf swing when using a driver. The COG displayed a consistent path across all right handed golfers in the backswing but there were differences in COG location at IMP. There was no clear reason given for this movement of COG and measures of performance, a golfer's postural kinematics or COP were not measured.

In summary, the biomechanical definition of posture echoes the coaches description as the degree of forward bend and lateral bend in a golfer's spine angle [Q8] and postural balance [Q12]. The golf coaches believed that maintaining a constant trunk angle throughout the swing, from TA through to IMP, would create consistent club position [Q10-11]. However, this perception cannot be fully supported by the literature as there are conflicting results and the pattern in trunk flexion throughout the whole swing has not been formally investigated. Previous studies have used ball velocity or clubhead velocity as measures of performance therefore the effect of trunk flexion on other measures of performance, such as shot accuracy and consistency, have not been fully investigated.

Trunk lateral bending was referred to by only a few coaches when discussing posture during the golf swing [Q9]. The contrasting literature, lack of performance related studies and minimal mention by coaches suggests that trunk lateral bend requires further investigation. Interestingly, the coaches alluded to the dependence of body rotations on posture [Q10], which is partially supported by the clinical study of Edmondston et al. [34].

The coaches in the perception study also identified the importance of postural balance and discussed the idea in terms of positioning a golfer's COG correctly [Q13] and their weight transfer [Q14], which echoes the biomechanical definition of static and dynamic balance. Often the coaches would make reference to a golfer's postural kinematics as a 
means of creating a balanced position throughout the swing [Q16]. However, the relationship between COG, COP and postural kinematics has not been investigated in the literature.

\section{BODY ROTATION}

When discussing posture the coaches also referred to the key technical parameter 'Body Rotation' [Q13]. Several terms were used to communicate the idea of body rotation including 'Core', 'Upper Torso,' 'Trunk', 'Shoulders', 'Hips' and 'Pelvis'. Nevertheless, the most common terms used were Shoulder and Hip rotations as these were deemed the most appropriate words to communicate clearly with the golfers during coaching sessions. Due to the various terms used to describe Body rotation, the terms trunk rotation and pelvis rotation will be used to aid clarity in this paper.

The rotation of the trunk and pelvis was referred to throughout the swing. The coaches believed that the rotation of the trunk and pelvis during the backswing was an opportunity to generate a powerful, repeatable and simple swing by producing torque or energy, which could then be transferred to the ball at impact.

...if there was minimal rotation...you're not going to be able to create as big torque in the backswing, create as much pressure in your right leg, therefore, you're not going to be able to shift that back across through into your left side and transfer that energy back through your arms and your club. [Q16]

I want the club to come down...I don't particularly want that to be fine movement with hands and arms...that can vary enormously...whereas hip turn can't vary as much. [Q17]

Only a selection of coaches offered preferences for the degree of rotation they would like to see during the swing, whereas other coaches commented that the degree of rotation was golfer specific, depending on elements such as a golfer's degree of flexibility.

[At] impact we're looking for the hips to be more turned open than the shoulders, within about 10 degrees...40 degrees with the hips and 35 to 30 degrees with shoulders is fine, as long as we've got the right tilts and right shifts into the left side. [Q18]

It was also recognised that body rotations would be influenced by movements within other planes and should not be disregarded:

Pelvic rotation... [is] rotation around its mid axis...but it doesn't just rotate...it shifts, it turns, it tilts as well so it's not simple rotation. [Q19]

The coaches believed that the separation between the trunk and pelvis was more important than the independent rotations of the segments. Many of the coaches spoke about the 'Disassociation', 'Resistance', 'Storing power' or 'Separation' between the trunk and pelvis segments. Others used the coined term 'X-factor' to describe the relationship between the trunk and pelvis rotations.

...coil on the backswing, storing power onto the right side...the initial movement of the hips beginning the downswing...you can see as the left foot, pulls the rest of the body through so the hips pull through the abs, the abs pull the chest and it all comes through and the big disassociation you can get between the hips and the shoulders, the more power. [Q20]

You get a good golfer who is stable...there will be a big difference between the hips and shoulders at the top of the backswing...that is one of the key factors of powerful golf swings, but it's not the key factor, the ability is to be able to separate the hips on the way down from the upper torso and then ... close that gap down as quick as we possibly can. [Q21]

In the golf biomechanical literature, body rotation, typically quantified by axial rotation of the central body segments, has been widely investigated and linked to performance outcomes, such as clubhead velocity $[19,38,39]$. Many studies have reported pelvis and trunk axial rotational angles at various stages of the swing including at TA, TB, IMP, middownswing, last 4oms prior to impact as well as the peak magnitudes. Hume et al. [8] reported trunk axial rotation of $78-102^{\circ}$ and pelvis axial rotation of $47^{-55^{\circ}}$ at TB depending on golfer ability and the club being used and also suggested that trunk flexion, lateral bend and knee angles should be observed as they could influence axial rotation.

Several authors have suggested that the separation between pelvis and trunk axial rotation (i.e. X-factor) was more important for power generation $[19,27]$. Chu et al. [27] reported that X-factor at TB explained approximately $25 \%$ of ball velocity with a driver and the authors suggested that golfers should focus on increasing separation between trunk and pelvis rotation in order to increase ball velocity. Maximum X-factor during the downswing was shown to strongly correlate with clubhead linear velocity at IMP ( 74\%) [40] and a moderate correlation was found between ball velocity using a driver and X-factor at TB $(\sim 30 \%)$. The authors concluded that $\mathrm{X}$-factor at TB and downswing maximum contributed to the rotation velocities of the upper torso which, in turn contributed to increased club and ball velocity.

The difference in X-factor between TB and downswing maximum value (termed $\mathrm{X}$-factor stretch), has been suggested as more important than the maximum X-factor alone [41]. The greater X-factor stretch (mean $13.4^{\circ}$ ) in highly skilled golfers (handicap < o) compared to a lower skilled golfer (handicap > 15) (mean 0.5 $5^{\circ}$ ) was considered to contribute to the greater shot distance for the higher skilled golfers [41]. It is important to note that the differences in how TB is defined could affect the value of X-factor at this part in the swing and subsequent X-factor stretch calculations. The rate of stretch and recoil describes the speed with which the trunk and pelvis separate and align providing a measure of rotational power [8]. Golfers with greater driving distance are suggested to display greater maximum rates of recoil in the downswing [22]. Nevertheless, there are limited studies that have investigated this idea further. The proposed mechanism for increased separation between trunk and pelvis and the timings of rotations was due to a stretch-shorten cycle within the spinal rotator muscles, leading to increased trunk acceleration and in turn increased club acceleration $[36,41]$. In contrast, studies on female golfers have not provided support for the stretch-shortening mechanism of trunk muscles during the downswing for increasing clubhead linear velocity [42].

The repeatability of rotational parameters have also been investigated [39,43-45]. Peak trunk axial rotation has been 
shown to have lower variability as shot intensity increased, while peak pelvis rotation repeatability was greater than trunk rotation repeatability across all shot intensities [39]. The rotational parameters at IMP displayed larger coefficient of variation (COV) than the peak values, which may be a consequence of consistently identifying the IMP position or how COV is defined. Horan et al. [44] examined movement variability of rotational parameters using standard deviations (SD) at key stages of the swing (TB, mid-downswing, IMP) and using spanning sets across continuous phases of the swing in male and female golfers. Female golfers were reported to have greater axial rotation variability for the pelvis at mid-downswing and IMP and trunk at IMP than males. However, the authors could not explain these differences in variability.

The majority of previous studies calculated trunk and pelvis axial rotation as $2 \mathrm{D}$ projection angles. These methods include simply using marker positions (e.g. acromion and anterior superior illiac spine (ASIS) markers, to define trunk and pelvis segment vectors [36]. Two-dimensional axial rotation angles are then calculated by projecting the vectors onto the global co-ordinate system horizontal plane. The X-factor calculated by the $2 \mathrm{D}$ projection method would be the angle between the projected pelvis and trunk vectors; however, limitations have been identified with this method. In reality, the golfer rotates about a flexed trunk (2D trunk $\left.\sim 30^{\circ}[43]\right)$ and projecting the trunk vector onto the global co-ordinate system horizontal plane could lead to errors in axial rotation angle measurements. Recognising that the $2 \mathrm{D}$ projection angles do not account for the six degrees of freedom of golf swing motion [46] more recent studies have used ${ }_{3} \mathrm{D}$ measurements to calculate trunk and pelvis axial rotation $[21,47,48]$. However, there has not been a direct comparison of X-factor magnitude between $2 \mathrm{D}$ projection methods and $3 \mathrm{D}$ measurement methods until recently [49,50]. Both studies reported statistically significant differences in X-factor values between the different computation methods. In particular, Kwon et al. [50] reported larger maximum X-factor values when using the $2 \mathrm{D}$ projection method compared to $3 \mathrm{D}$ angles $\left(59.1 \pm 10.6^{\circ}\right.$ vs. $\left.55.7 \pm 10.0^{\circ}\right)$.

In summary, the separation between trunk and pelvis was viewed as more important than rotations of individual segments by golf coaches [Q20-21], which is in agreement with most of the biomechanical literature. Some coaches alluded to other important aspects of X-factor, such as rate of recoil [Q21]; there have been few studies to investigate this premise. Although coaches were largely concerned with body rotation they did not discount the effect of movement in other directions such as shifts or translation [Q18-19], which is sometimes discounted when biomechanical studies report $2 \mathrm{D}$ methods. This will require determination of the most appropriate methodologies to account for both rotations and translations. Coaches would also link body rotation to powerful, repeatable and simple swings [Q17]. Although, body rotation varied at discrete stages, the variability throughout the swing and across golfers needs further investigation.

\section{CONCLUSION}

The purpose of this study was to identify the key technical parameters that high-level golf coaches associate with a successful golf swing and to compare them to current golf biomechanical literature to identify areas for future golf biome- chanical research. Five key technical parameters emerged from the inductive analysis which were, 'Club Motion', 'Posture', 'Body Rotation', 'Sequential Movement of Body Segments' and 'Arm and Wrist Action'. Coaches were keen to analyse the golf swing as a whole or during different phases of the movement (e.g. initial downswing), which the current golf biomechanical analysis methods do not readily report. Posture and body rotation were the most common themes discussed by golf coaches and were often discussed together. Posture was identified as a key biomechanical parameter in the literature; however, there were limitations with the methodologies used to measure trunk flexion and lateral bend and the relationship between postural kinematics and postural balance was not examined. Body rotation was also related to posture in the biomechanical literature, however again methodological limitations make it difficult to examine the coupled movement. The separation between pelvis and trunk axial rotation were viewed as key to producing powerful swings but the mechanisms are not fully understood. The results of this study have led to the formation of two prevailing research questions; Are existing biomechanical data collection and analysis methods appropriate for measuring posture and body rotation of the golf swing?; and How can we biomechanically analyse posture and body rotation for individual golfers and throughout the swing to further understand their relationship with performance?

\section{REFERENCES}

1. Sherman, CA., Sparrow, WA., Jolley, D. and Eldering, J., Coaches' Perceptions of Golf Swing Kinematics, International Journal of Sport Psychology, 2001,31, 257-70.

2. Jones, RL., Hughes, M. and Kingston, K., An Introduction to Sports Coaching: From Science and Theory to Practice, Routledge, Abingdon, 2008

3. Thompson, A., Bezodis, I.N. and Jones, RL., An InDepth Assessment of Expert Sprint Coaches' Technical Knowledge, Journal of Sports Sciences, 2009, 27(8), 855-61.

4. Irwin, G., Hanton, S. and Kerwin, D., The Conceptual Process of Skill Progression Development in Artistic Gymnastics, Journal of Sports Sciences, 2005, 23(10),1089-99.

5. Greenwood, D., Davids, K. and Renshaw, I., How Elite Coaches' Experiential Knowledge Might Enhance Empirical Research on Sport Performance, International Journal of Sports Science and Coaching, 2012, 7(2), 411-22.

6. Schempp, P,G., Webster, C., Mccullick, B., Busch, C. and Sannen Mason, I., How the Best Get Better: an Analysis of the Self-Monitoring Strategies Used by Expert Golf Instructors, Sport, Education and Society, 2007,12(2),175-92.

7. Adlington, G., Proper Swing Technique and Biomechanics of Golf, Clinics In Sports Medicine, 1996,15(1),9-26.

8. Hume, P.A., Keogh, J. and Reid, D., The Role of Biomechanics in Maximising Distance and Accuracy of Golf Shots, Sports Medicine, 2005, 35(5), 429-49.

9. Lees, A., Biomechanical Assessment of Individual Sports for Improved Performance, Sports Medicine, 1999, 28(5), 299-305.

10. Cote, J., Salmela, J. and Russell, S., The Knowledge of High-Performance Gymnastic Coaches: Methodological Framework, Framework, 1995, 65-75.

11. Jones, R., Bezodis, I. and Thompson, A., Coaching Sprinting: Expert Coaches' Perception of Race Phases and Technical Constructs, International Journal of Sports Science and Coaching, 2009, 4(3), 385-96. 
12. Patton, M., Qualitative Research and Evaluation Methods. 3rd Edn., Sage Publications, London, 2002.

13. Meyer, B.B. and Wenger, M.S., Athletes and Adventure Education: An Empirical Investigation., International Journal of Sport Psychology, 1998, 29(3), 243-66.

14. Kvale, S., Doing Interviews, Sage Publications, London, 2007.

15. Roberts, J., Jones, R., Harwood, C. and Mitchell, S., Human Perceptions of Sports Equipment Under Playing Conditions Human Perceptions of Sports Equipment Under Playing Conditions, Journal of Sports Sciences, 2001, 485-97.

16. Walker, D. and Myrick, F., Grounded Theory: An Exploration of Process and Procedure, Qualitative Health Research, 2006,16(4), 547-59.

17. Corbin, J. and Strauss, A., Basics of Qualitative Research: Techniques and Procedures For Developing Grounded Theory, Sage Publications, London, 2008.

18. Zheng, N., Barrentine, SW., Fleisig, GS. and Andrews, JR., Kinematic Analysis of Swing in Pro and Amateur Golfers, International Journal of Sports Medicine, 2008, 29(6), 487-93.

19. Burden, A.M., Grimshaw, P.N. and Wallace, E.S., Hip and Shoulder Rotations During the Golf Swing of Sub-10 Handicap Players., Journal of Sports Sciences, 1998,16(2),16576.

20. Coleman, S. and Rankin, A.A., Three-Dimensional Examination of the Planar Nature of the Golf Swing., Journal of Sports Sciences, 2005, 23(3), 227-34.

21. Wheat, J.S., Vernon, T. and Milner, C.E., The Measurement of Upper Body Alignment During the Golf Drive, Journal of Sports Sciences, 2007, 25(7), 749-55.

22. Neal, R., Introducing the Rate of Recoil of A Golf Swing [Internet], Golf Biodynamics. 2008. Available from: http://www.totalgolfanalysis.co.uk/download/articlerateofrecoil.pdf

23. Coffey, N., Harrison, A.J., Donoghue, O.A. and Hayes, K., Common Functional Principal Components Analysis: A New Approach to Analyzing Human Movement Data., Human Movement Science, 2011, 30(6),1144-66.

24. Donà, G., Preatoni, E., Cobelli, C., Rodano, R. and Harrison, A.J., Application of Functional Principal Component Analysis in Race Walking: an Emerging Methodology, Sports Biomechanics, 2009, 8(4), 284-301.

25. Lynn, S.K., Noffal, G.J., Wu, W.F.W. and Vandervoort, A.A., Using Principal Components Analysis to Determine Differences in 3D Loading Patterns Between Beginner and Collegiate Level Golfers, Journal of Golf Science, 2012, 25-41.

26. Winter, D., Human Balance and Posture Control During Standing and Walking, Gait \& Posture, 1995,3(4),193214.

27. Chu, Y., Sell, T. and Lephart, S., The Relationship Between Biomechanical Variables and Driving Performance During the Golf Swing., Journal of Sports Sciences, 2010, 28(11),1251-9.

28. McTeigue, M, Lamb, S.R., Mottram, R. and Pirozzolo, F., Spine and Hip Motion Analysis During the Golf Swing, Science and Golf II: Proceedings of The World Scientific Congress of Golf, 1994, 50-58.

29. Miura, K.N., Accelerating and Decelerating Phases of the Wrist Motion of the Golf Swing, In The Engineering of Sport: Design and Development, 2001.

30. Zheng, N., Barrentine, S.W., Fleisig, G.S. and Andrews, J.R., Swing Kinematics For Male and Female Pro Golf- ers., International Journal of Sports Medicine, 2008,29(12),965-70.

31. Lindsay, D.M. and Horton, J.F., Trunk Motion of Male Professional Golfers Using Two Different Golf Clubs, Journal of Applied Biomechanics, 2002,18, 366-73.

32. Perry, S.D., Radtke, A. and Goodwin, C.R., Influence of Footwear Midsole Material Hardness On Dynamic Balance Control During Unexpected Gait Termination, Gait \& Posture, 2007, 25(1), 94-8.

33. Ball, K. and Best, R., Different Centre of Pressure Patterns Within the Golf Stroke I: Cluster Analysis, Journal of Sports Sciences, 2007, 25(7), 757-70.

34. Ball, K. and Best, R., Centre of Pressure Patterns in the Golf Swing: Individual-Based Analysis, Sports Biomechanics / International Society of Biomechanics in Sports, 2012,11(2),175-89.

35. Ball, K. and Best, R., Different Centre of Pressure Patterns Within the Golf Stroke II: Group-Based Analysis, Journal of Sports Sciences, 2007, 25(7), 771-9.

36. Burden, A., Grimshaw, P. and Wallace, E., Hip and Shoulder Rotations During the Golf Swing of Sub-10 Handicap Players, Journal of Sports Sciences, 1998,16(2),165-76.

37. Edmondston, S., Aggerholm, M., Elfving, S., Flores, $\mathrm{N} ., \mathrm{Ng}, \mathrm{C}$. and Smith, R., Influence of posture on the range of axial rotation and coupled lateral flexion of the thoracic spine, Journal of Manipulative and Physiological Therapeutics, 2007, 30(3), 193-9.

38. Egret, C., Nicolle, B., Dujardin, F., Weber, J. and Chollet, D., Kinematic Analysis of the Golf Swing in Men and Women Experienced Golfers., International Journal of Sports Medicine, 2006, 27(6), 463-7.

39. Meister, D.W., Ladd, A.L., Butler, E.E., Zhao, B., Rogers, A.P. and Ray, C.J., Rotational Biomechanics of the Elite Golf Swing: Benchmarks For Amateurs, Journal of Applied Biomechanics, 2011, 27(3), 242-51.

40. Myers, J., Lephart, S., Tsai, Y-S., Sell, T., Smoliga, J. and Jolly, J., The Role of Upper Torso and Pelvis Rotation in Driving Performance During the Golf Swing., Journal of Sports Sciences, 2008, 26(2), 181-8.

41. Cheetham, P. and Martin, P., The Importance of Stretching the "X-Factor " in the Downswing of Golf: The " X-Factor Stretch ," Golf Magazine, 2001,1-11.

42. Brown, S., Nevill, A., Monk, S., Otto, S., Selbie, S. and Wallace, E., Determination of the Swing Technique Characteristics and Performance Outcome Relationship in Golf Driving For Low Handicap Female Golfers., Journal of Sports Sciences, 2011, 29(14), 1483-91.

43. Bradshaw, E., Keogh, J., Hume, P., Maulder, P., Nortje, J. and Marnewick, M., The Effect of Biological Movement Variability On the Performance of the Golf Swing in High- and Low-Handicapped Players, Research Quarterly For Exercise and Sport, 2009, 8o(2),185-96.

44. Horan, S., Evans, K. and Kavanagh, J., Movement Variability in the Golf Swing of Male and Female Skilled Golfers, Medicine and Science in Sports and Exercise, 2011, 43(8), 1474-83.

45. Tucker, C., Is Movement Variability Relevant For the Elite Golfer? A Biomechanical and Modelling Perspective, $\mathrm{PhD}$ Thesis, University of Limerick, 2012.

46. Horan, S., Evans, K., Morris, N. and Kavanagh, J., Thorax and Pelvis Kinematics During the Downswing of Male and Female Skilled Golfers, Journal of Biomechanics, 2010,1-7. 
47. Horan, S.A. and Kavanagh, J.J., The Control of Upper Body Segment Speed and Velocity During the Golf Swing, Sports Biomechanics / International Society of Biomechanics in Sports, 2012,11(2),165-74.

48. Joyce, C., Burnett, A. and Ball, K., Methodological Considerations For the $3 \mathrm{D}$ Measurement of the X-Factor and Lower Trunk Movement in Golf., Sports Biomechanics, 2010, 9(3), 206-21.

49. Brown, S.J., Selbie, W.S. and Wallace, E.S., The XFactor: an Evaluation of Common Methods Used to Analyse Major Inter-Segment Kinematics During the Golf Swing, Journal of Sports Sciences, 2013, 31(11), 1156-63.

50. Kwon, Y-H., Han, K.H., Como, C., Lee, S. and Singhal, K., Validity of the X-Factor Computation Methods and Relationship Between the X-Factor Parameters and Clubhead Velocity in Skilled Golfers, Sports Biomechanics, 2013,1-1 